\title{
Freeform Surface Lens Design Using Genetic Algorithm with Acrylic Material for Reducing Aberrations in Multifocal Artificial Intraocular Lens to Enhance Image Sensing Quality
}

\author{
Chih-Ta Yen ${ }^{1 *}$ and Shih-Cyuan Jin ${ }^{2}$ \\ ${ }^{1}$ Department of Electrical Engineering, National Taiwan Ocean University, Keelung City 202301, Taiwan \\ ${ }^{2}$ Department of Electrical Engineering, National Formosa University, Yunlin County 632, Taiwan
}

(Received May 25, 2021; accepted December 2, 2021)

Keywords: intraocular lens (IOL), third-order aberrations, freeform surface lens, genetic algorithm (GA)

A complex intraocular lens (IOL) design involving numerous uncertain variables is proposed. We integrated a genetic algorithm (GA) with a freeform surface lens by using CODE V optical design software to design a multifocal IOL for the human eye. We mainly used a freeform surface lens of acrylic material in the initial crystalline state to enhance image sensing quality; therefore, we used the internal human eye model in the software. The proposed optimization algorithm employs a GA method to optimally simulate the focusing function of the human eye; in this method, the thickness and curvature of the anterior lens and the posterior part of the IOL were varied. We performed a comparison of the proposed GA-designed IOLs and those designed using a CODE V built-in optimization algorithm for the hyperopia 300 degree condition of the human eye. When the pupil entrance was $6 \mathrm{~mm}$, the proposed IOL design improved the RMS of the spot diagram by approximately 11.99 and $10.65 \%$ for the human eye to object distances of $35 \mathrm{~cm}$ and infinity, respectively. Moreover, the modulation transfer function (MTF) was improved by approximately 5.06 and $15.06 \%$ for distances of $35 \mathrm{~cm}$ and infinity at a spatial frequency of 15 cycles $/ \mathrm{mm}$, respectively.

\section{Introduction}

Intraocular lens (IOL) implants have been around for more than 71 years. ${ }^{(1)}$ The effect of the shape of a poly(methyl methacrylate) IOL on the optical performance of pseudophakic eyes has been investigated. Image quality criteria mainly include wave aberrations, spot diagrams, longitudinal aberrations, and the modulation transfer function (MTF). For eyes with corneal asphericities, accounting for most of the population, the optimum lens form for on-axis vision is close to plano-convex, with the more curved surface facing the cornea. ${ }^{(2)}$

The human eye suffers from a number of specific optical aberrations. The optical quality of the eye is limited by optical aberrations, diffraction, and scatter. Aberrations of the human eye play a major role in degrading retinal image quality. ${ }^{(3-7)}$ A closed-form solution determining the personalized required shape of a new IOL that is able to remove the spherical aberration and coma of a pseudophakic eye has been reported. ${ }^{(8)}$ The proposed analytical method, which is *Corresponding author: e-mail: chihtayen@gmail.com https://doi.org/10.18494/SAM3556 
within the framework of the Seidel theory of third-order optical aberrations, considers corneal conicities, the fourth-order aspherical surface of the intraocular optics, the pupil-shift effect, and the ocular kappa angle.

Phacoemulsification with IOL implantation has been a common treatment for cataract patients. With rising demand among the public, new technologies for lens design have emerged to minimize intraocular aberrations, improving visual quality. Reference 9 systematically reviews the development of materials applied in lens manufacturing, the different categories of IOLs, and their design principles. High-order aberrations have been measured using a Hartmann-Shack aberrometer with wavefront aperture diameters of 4.0 and $6.0 \mathrm{~mm} .^{(10)}$

The eye has many innate adaptations that minimize optical aberrations. In most normal young eyes, the magnitude of aberrations of the cornea is significantly larger than that of the whole eye, indicating a significant role of the crystalline lens in compensating corneal aberrations. However, owing to geometrical and structural changes, this ocular compensation becomes disturbed under different anterior segment conditions, such as keratoconus, presbyopia, and cataract. Keratoconus progressively degrades the corneal shape and, consequently, vision in adolescence, with a prevalence of $0.05 \%$ in the general population. ${ }^{(11)}$ Standard IOLs are mostly designed for long-distance vision. Although accommodative IOLs have been proposed, the current designs only provide a modest range of accommodation in the best-case scenario. We describe here a new optical design providing a wide range of potential accommodation change that could be incorporated in an IOL. ${ }^{(12)}$ The effect of the IOL shape on the off-axis performance of model pseudophakic eyes was investigated. In this investigation, the optimum lens shapes for a $10^{\circ}$ off-axis position ranged from one with an off-axis angle of nearly $10^{\circ}$ to nearly the reverse shape, i.e., with the more curved surface facing the retina. ${ }^{(13)}$

Presbyopia is the age-related loss of accommodative function and the reason that almost all people need reading glasses, bifocals, or some other optical correction after the age of 45 years. Presbyopia-correcting IOLs have been developed to overcome the need for eyeglasses or contact lenses for high-resolution near vision (i.e., reading) in adulthood. These presbyopia-correcting IOLs can be classified as either accommodative or multifocal. ${ }^{(14)}$ In Ref. 15, a design for a diffractive multifocal IOL was presented with the primary goal of compensating for as much of the effect of chromatic aberration as possible. As longitudinal chromatic aberrations (LCAs) due to diffraction and refraction act in opposite directions, LCAs can in principle be compensated. However, rules to create an achromatic lens based on a refractive-diffractive lens are complex because of the topology, dioptry, Abbe number, and so forth. In this study, we investigate the effects of the wavelength on the through-focus MTF and diffraction efficiency for different pupil apertures and diffractive IOLs. The material of the IOLs is hydrophobic acrylate. A multifocal IOL with a diffractive surface on the anterior surface and an aspherical surface is designed. ${ }^{(16)}$

The main objective of this study was to devise a method to design IOLs for correcting hyperopia. Because of technological advancements, people frequently use mobile phones and other computer, communication, and consumer electronic products. The use of these products causes the focusing function of the human eye lens to gradually deteriorate and the focal point to shift backward, causing hyperopia. The older a person is, the easier it is to lose the original flexibility of the crystalline lens. This loss of flexibility results from the accumulation of protein and produces symptoms of presbyopia. The general treatment prescribed is the use of presbyopic 
glasses. However, extra glasses inconvenience people. Therefore, researchers began exploring ways to improve human vision without using glasses. The corneal or crystalline lens is located in the interior of the human eye and is therefore difficult to correct. In the present study, a method was devised for designing a suitable IOL for different conditions of the human eye. First, we utilize the CODE V internal human eye model to simulate an imperfect human eye and use an optical design scheme involving an aspherical lens to design the IOL. Second, a genetic optimization algorithm is used to achieve the best results for human image qualities. Because it is difficult to change the corneal or crystalline lens in the human eye, it is crucial to use a welldesigned artificial IOL for imperfect human eyes. Hence, an IOL design with good imaging performance can eliminate the use of external lenses to correct vision. The rest of this paper is organized as follows. Section 2 provides an overview of the methodology of the optical IOL design. In Sect. 3, we analyze the theory and formulas and discuss the proposed simulation performance. In Sect. 4, we discuss the optical performance after applying the proposed genetic algorithm (GA) method. Section 5 lists the relative improvements of our simulations when applying the proposed method. Section 6 summarizes the paper.

\section{Methodology of Optical IOL Design}

In this study, we used a freeform surface lens and a GA to design a freeform surface IOL to reduce aberrations in an imperfect human eye. The design consisted of two steps. (1) We used the internal human eye model of Liou and Brennan in CODE V to model the human eye lens patterns as the human eye model basis in our study. ${ }^{(17)}$ In the freeform surface technique used to simulate a human eye IOL, parameters such as diffraction grating parameters, curvature, and thickness are adjusted to modify the original surface of the IOL to adjust the focal length of the objective. (2) The GA scheme was used to optimize the IOL to reduce aberrations and improve the MTF. The CODE V software enables GA optimization to be performed in a manner that minimizes the error function, thereby helping achieve more accurate focusing.

\section{Theory and Formula Analysis}

\subsection{Initial state of optical human eye model}

We used the internal optical human eye model in CODE V, which was proposed by Liou and Brennan in 1997. However, this model is mainly based on the nonspherical development; therefore, to design an IOL, it was essential to change its aspherical curvature and thickness. Table 1 lists the initial specifications of the crystalline lens of the human eye in the IOL design. The 2D layout of the human eye crystalline lens is shown in Fig. 1. Table 2 presents the thirdorder aberrations of the initial CODE $\mathrm{V}$ design.

The simulation is mainly based on the human eye in the hyperopia 300 degree condition. ${ }^{(18)}$ Through the selection and matching of lens data using the GA, we find the optimal parameters of a Fresnel lens and the diffraction grating without adjusting the length of the IOL to realize a multifocal IOL. The fourth lens is replaced by the parameters of the Fresnel plane base plus the diffraction grating. Since the design of a freeform surface lens is more complicated, two zoom 
Table 1

Initial specifications of the crystalline lens of the human eye.

\begin{tabular}{ll}
\hline \multicolumn{2}{c}{ Initial conditions of design } \\
\hline Anterior radius of crystalline lens & $0 \mathrm{~mm}$ \\
Posterior radius of crystalline lens & $0.0806 \mathrm{~mm}$ \\
Anterior thickness of crystalline lens & $1.59 \mathrm{~mm}$ \\
Posterior thickness of crystalline lens & $2.43 \mathrm{~mm}$ \\
\hline
\end{tabular}

Table 2

Third-order aberrations in the initial human eye model.

\begin{tabular}{lc}
\hline Third-order aberration & Value \\
\hline SA (spherical aberration) & -0.038380 \\
TCO (coma aberration) & -0.058892 \\
TAS (tangential surface astigmatism) & -0.698977 \\
SAS (sagittal surface astigmatism) & -0.500537 \\
PTB (third-order Petzval blur) & -0.401317 \\
DST (distortion) & -0.422739 \\
\hline
\end{tabular}

Table 3

Initial lens parameters for a distance of $35 \mathrm{~cm}$.

\begin{tabular}{lcccc}
\hline Surface\# & Surface type & Y radius & Thickness & Glass \\
\hline \#Object & Sphere & 0 & 350 & - \\
$\# 1$ & Conic & 7.77 & 0.5 & 1.376 \\
$\# 2$ & Conic & 6.4 & 3.16 & 1.336 \\
\#Stop & Sphere & 0 & 0 & 1.336 \\
\#4 & Fresnel & 0.0806 & 1.59 & 1.493313 \\
$\# 5$ & Sphere & 0 & 2.43 & 1.493313 \\
\#6 & Conic & -0.1235 & 16.27 & 1.336 \\
\#Image & Sphere & -11 & 0 & 1.336 \\
\hline
\end{tabular}

Table 4

Initial lens parameters for an infinite distance.

\begin{tabular}{lcccc}
\hline Surface\# & Surface type & Y radius & Thickness & Glass \\
\hline \#Object & Sphere & 0 & Infinity & - \\
$\# 1$ & Conic & 7.77 & 0.5 & 1.376 \\
$\# 2$ & Conic & 6.4 & 3.16 & 1.336 \\
\#Stop & Sphere & 0 & 0 & 1.336 \\
$\# 4$ & Fresnel & 0.0806 & 1.59 & 1.493313 \\
$\# 5$ & Sphere & 0 & 2.43 & 1.493313 \\
$\# 6$ & Conic & -0.1235 & 16.27 & 1.336 \\
\#Image & Sphere & -11 & 0 & 1.336 \\
\hline
\end{tabular}

segments with short and long distances from the human eye to the object are used in the simulations in this study. Tables 3 and 4 show the initial lens data before optimization for distances of $35 \mathrm{~cm}$ and infinity, respectively. initial crystalline lens. ${ }^{(17)}$

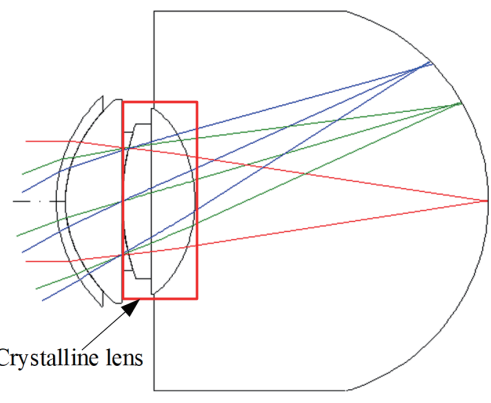

Fig. 1. (Color online) 2D layout diagram of the 
The simulations are based on the human eye model of hyperopia 300 degree. Figure 2 shows a 2D plot of the initial human eye with hyperopia 300 degree. Figures 3 and 4 respectively show the MTF plot and spot diagram of the initial human eye with hyperopia 300 degree.

\subsection{Freeform surface lens design}

Because the design scheme of a freeform surface lens is complicated, we choose two zoom segments, one near and one far, in the simulations. In the design of the freeform surface lens, we change the parameters on the aspheric and conic coefficients to achieve Fresnel zigzag shapes. In the Fresnel lens design, the parameters defined for the fourth, sixth, and eighth orders are

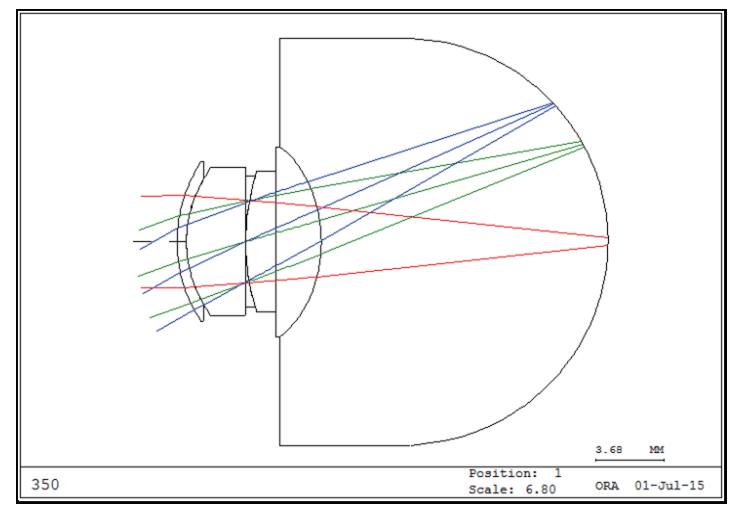

Fig. 2. (Color online) 2D plot of initial human eye with hyperopia 300 degree.

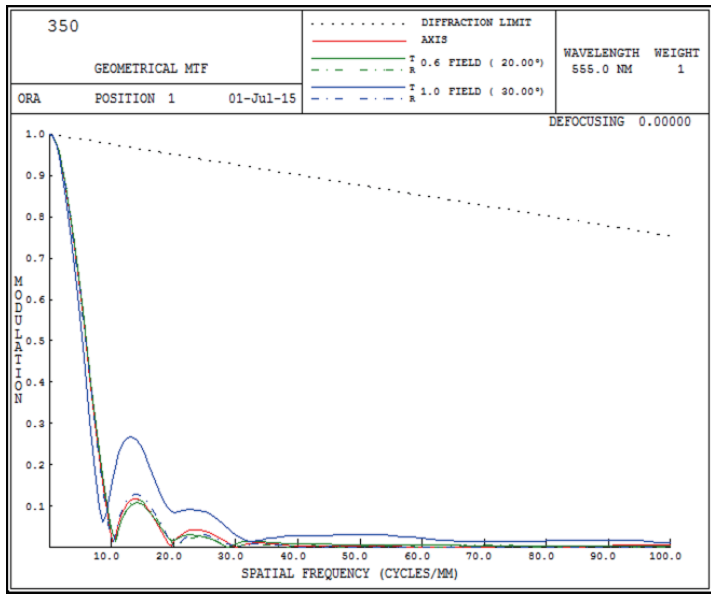

Fig. 3. (Color online) MTF plot of initial human eye with hyperopia 300 degree.

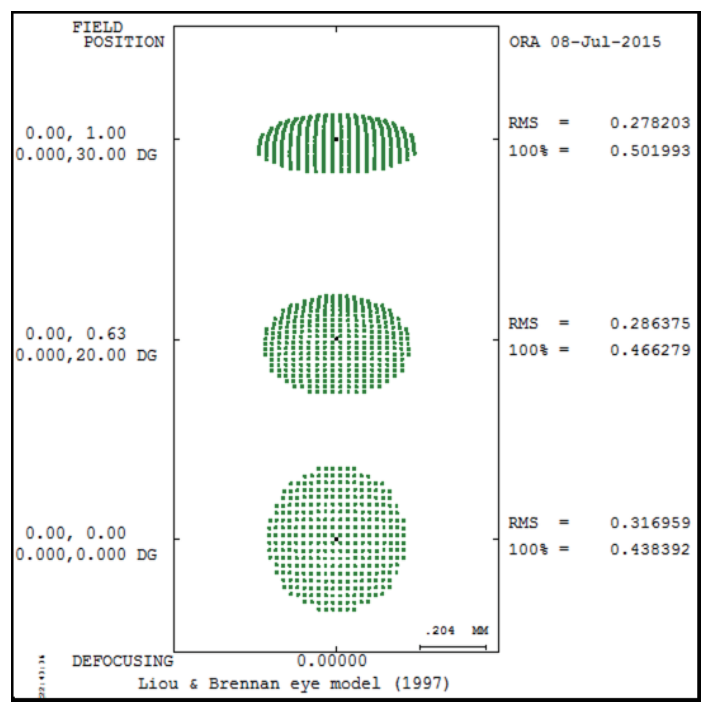

Fig. 4. (Color online) Spot diagram of initial human eye with hyperopia 300 degree. 
$4.785768 \times 10^{-9},-6.65579 \times 10^{-20}$, and $6.01612 \times 10^{-13}$, and the maximum discrete area, the area sagittal height, and the center of the area sagittal height are $10,0.125$, and 0.125 , respectively. CODE V software has the ability to search for fringes and raster spacing on a curved surface by using the diffraction characteristics of the grating to modify the direction of light. Therefore, in the multi-focus design, the Fresnel lens characteristic is mainly used for the setting of the curved surface of the lens, and the setting of the diffraction order is used to determine the direction of light.

We use the Fresnel plane substrate surface in CODE V software to find the optimal number of orders to substitute into Eq. (1). The verticality of a groove $z$ is given as

$$
z=\frac{c r^{2}}{1+\sqrt{1-(1+k) c^{2} r^{2}}}+Q r^{2}+A r^{4}+B r^{6}+\ldots+J r^{22},
$$

where $c$ is the curvature of the vertex, $k$ is the conic coefficient, and the parameters $Q$ to $J$ are the orders.

We apply the refraction law of the Fresnel lens and the light direction controlled by the diffraction grating to realize a freeform IOL with multi-focus functions. The phase polynomial diffraction grating is used to find the optimal light direction, which is expressed as

$$
\begin{gathered}
\eta_{m}=\left\{\frac{\sin [\pi(\beta-m)]}{\pi(\beta-m)}\right\}^{2}, \\
\beta=\frac{d\left(n_{1} \cos \theta_{1}-n_{2} \cos \theta_{2}\right)}{\lambda},
\end{gathered}
$$

where $m$ is the diffraction order, $d$ is the blaze depth, $n_{1}$ is the front surface refractive index, $n_{2}$ is the back surface refractive index, $\lambda$ is the light wavelength, $\theta_{1}$ is the angle of incidence, and $\theta_{2}$ is the angle of diffraction. Figure 5 is a schematic diagram of a diffraction grating.
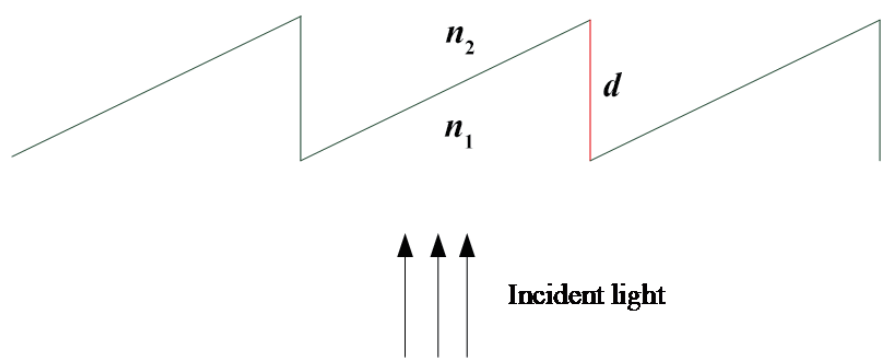

Fig. 5. (Color online) Schematic diagram of diffraction grating. 
The equation used to find the parameter $d$ is

$$
d=\frac{m \lambda}{\left(n_{1}-n_{2}\right)}
$$

In this study, we mainly use a Fresnel lens as a freeform surface lens for realizing a multifocus function. We also incorporate the theories of diffraction and refraction in the optical design. We choose a polynomial with rotational symmetry for the grating to design a concentric lens to realize a multifocal IOL.

\subsection{GA optimization technology}

The concept of the GA is derived from Darwin's concept of evolution, that is, the theory of natural selection, which postulates the survival of the fittest. The less fit members of a population are eliminated through the reproduction process; the resilience of individual organisms makes it relatively easy for them to find a good mate with which to produce the next generation. Over generations, organisms thus can adapt to the environment given stable growth conditions. ${ }^{(19-21)}$

The GA technique is applied to the optical design of the artificial IOL. The advantages of integrating the GA optimization process with the optical design are the obtention of the overall physical artificial crystal with better focusing function and higher flexibility. The proposed GA scheme is used to match and change the lens data, thus achieving better optical performance than that obtained with the CODE V built-in function. Simulation results showed that not only were the third-order aberrations reduced, but the DST, MTF, and spot size were also improved, leading to the accurate focusing of light on the retina. The IOL design mainly involves designing the curvature and thickness to improve the quality of vision. Therefore, the chromosomes in our GA represented the curvature and thickness, which randomly generated a new curvature and thickness gene according to the ethnic population. The calculation process used the fitness function to determine good vision qualities to facilitate the subsequent crossover and mutation mechanisms. The fitness function used for GA optimization was

$$
f i t(i)=\sum_{l=1}^{2} w_{1}\left|S A^{l}\right|+w_{2}\left|T A S^{l}\right|+w_{3}\left|S A S^{l}\right|,
$$

for $i=1,2, \ldots$, pop_size, where $l$ is the zoom section number and $w_{1}, w_{2}$, and $w_{3}$ are the weights of the fitness function. A sorting method was used for crossover and mutation to identify a suitable gene in the optical design; subsequently, in accordance with the roulette wheel probability, a fitness function was chosen for crossover and mutation. The selection probabilities of the roulette wheel diagram are shown in Fig. 6.

Finally, after crossover and mutation, the parameters of the resulting chromosomes are substituted into CODE V to design the IOL. A flowchart of the GA is shown in Fig. 7.

Table 5 shows the optimized cone coefficient, Y curvature, and thickness of the IOL after GA optimization, Table 6 shows the Fresnel lens parameters after GA optimization, and 


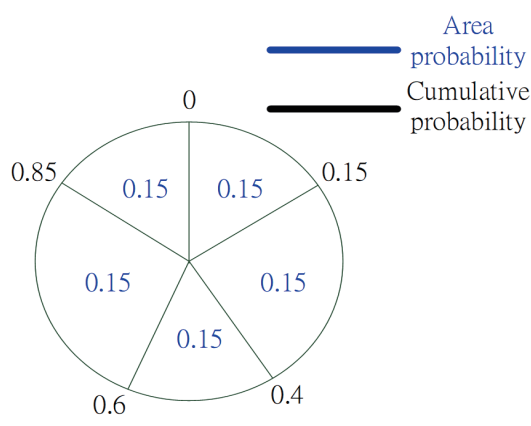

Fig. 6. (Color online) Schematic of a roulette wheel.

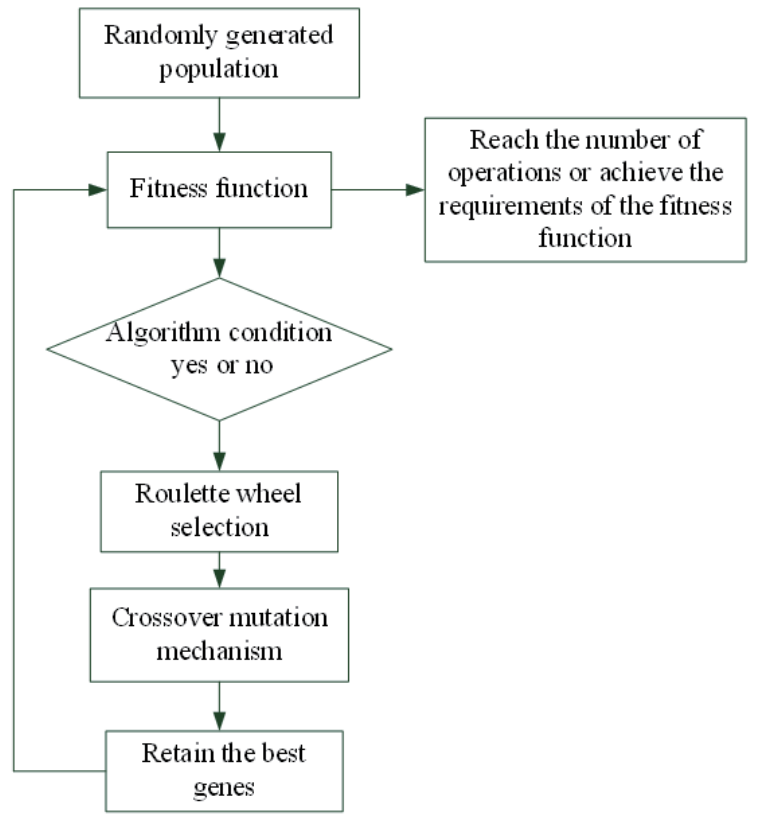

Fig. 7. (Color online) Flowchart of the GA used in the IOL design.

Table 5

Cone coefficient, Y curvature, and thickness after GA optimization.

\begin{tabular}{lccc}
\hline Surface \# & Cone coefficient & Y curvature & Thickness \\
\hline 4 & 21.9444 & 0.0672 & 1.2276 \\
5 & 0 & 0 & 2.8314 \\
6 & -3.7082 & -0.1388 & 15.27 \\
\hline
\end{tabular}

Table 6

Fresnel lens parameters after GA optimization.

\begin{tabular}{lcccccc}
\hline Surface \# & $\begin{array}{c}\text { Fourth-order } \\
\text { aspheric } \\
\text { coefficient }\end{array}$ & $\begin{array}{c}\text { Sixth-order aspheric } \\
\text { coefficient }\end{array}$ & $\begin{array}{c}\text { Eighth-order } \\
\text { aspheric coefficient }\end{array}$ & $\begin{array}{c}\text { Maximum } \\
\text { discrete area }\end{array}$ & $\begin{array}{c}\text { Area sagittal Central of area } \\
\text { height }\end{array}$ & $\begin{array}{c}\text { sagittal height } \\
\text { sagn }\end{array}$ \\
\hline 4 & 0.000374 & $-8.0464 \times 10^{-5}$ & $-1.7574 \times 10^{-5}$ & 10 & 0.125 & 0.125 \\
\hline
\end{tabular}

Tables 7 and 8 show the diffraction orders for different distances and the diffraction coefficients for different orders, respectively.

\section{Optical Performance Analysis}

\subsection{Third-order aberration analysis}

The GA criteria in the design are based on minimizing SA, TAS, and SAS to realize a freeform surface lens. Table 9 shows the third-order aberrations after GA optimization. 
Table 7

Diffraction orders for different distances.

\begin{tabular}{lc}
\hline Distance & Diffraction order \\
\hline $35 \mathrm{~cm}$ & 2 \\
Infinity & 8 \\
\hline
\end{tabular}

Table 8

Diffraction coefficients for different orders after GA optimization.

\begin{tabular}{lccc}
\hline Surface \# & $\begin{array}{c}\text { Fourth-order } \\
\text { diffraction coefficient }\end{array}$ & $\begin{array}{c}\text { Sixth-order diffraction } \\
\text { coefficient }\end{array}$ & $\begin{array}{c}\text { Eighth-order diffraction } \\
\text { coefficient }\end{array}$ \\
\hline 4 & $9.8673 \times 10^{-5}$ & $-1.2209 \times 10^{-5}$ & $5.515 \times 10^{-7}$ \\
\hline 6 & $-6.7728 \times 10^{-6}$ & $-5.1587 \times 10^{-7}$ & $5.8132 \times 10^{-8}$ \\
\hline
\end{tabular}

Table 9

Third-order aberrations after GA optimization.

\begin{tabular}{lcc}
\hline $\begin{array}{l}\text { SA } \\
(\text { CODE V })\end{array}$ & -0.063012 & -0.061338 \\
\hline $\begin{array}{l}\text { TCO } \\
(\text { CODE V })\end{array}$ & 0.132076 & 0.130273 \\
\hline $\begin{array}{l}\text { TAS } \\
(\text { CODE V })\end{array}$ & -0.418144 & -0.388390 \\
\hline $\begin{array}{l}\text { SAS } \\
(\text { CODE V })\end{array}$ & -0.386417 & -0.365586 \\
\hline $\begin{array}{l}\text { SA } \\
(\text { GA })\end{array}$ & -0.063012 & -0.060062 \\
\hline $\begin{array}{l}\text { TCO } \\
(\text { GA })\end{array}$ & 0.039395 & 0.042146 \\
\hline $\begin{array}{l}\text { TAS } \\
(\text { GA })\end{array}$ & -0.396602 & -0.368300 \\
\hline $\begin{array}{l}\text { SAS } \\
(\text { GA })\end{array}$ & -0.338056 & -0.319541 \\
\hline
\end{tabular}

\subsection{Two-dimensional plot analysis}

As shown in Fig. 8, the designed Fresnel lens shape has an irregular shape after GA optimization. This is because when light enters the human eye, the different refractive indices of different curved surfaces can allow the light from different distances to be accurately directed to the retina. Figure 9 shows a 2D plot of the human eye after GA optimization for different distances.

As shown in Figs. 10(a) and 10(c), the RMSs of the spot diagram are 0.0279325 and 0.024122 after CODE V and GA optimization for a distance of $35 \mathrm{~cm}$, respectively. For the infinite distance, the RMSs of the spot diagram are 0.0261005 and 0.023188 after CODE V and GA optimization, respectively. The results show that the GA-optimized performance is superior to that of the CODE V built-in algorithm. 


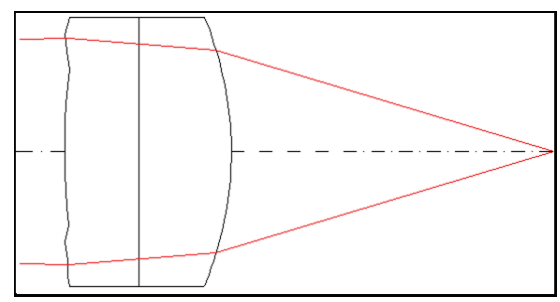

Fig. 8. (Color online) 2D plot of IOL after GA optimization.

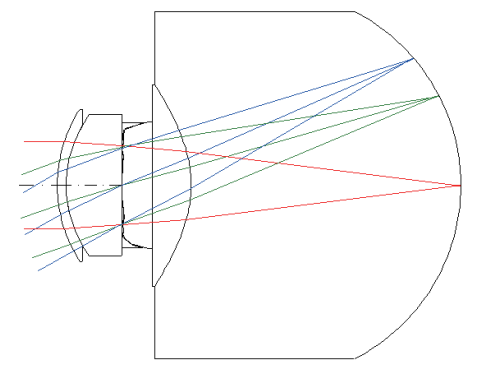

(a)

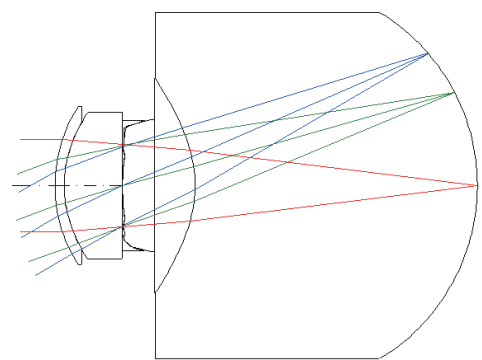

(b)

Fig. 9. (Color online) 2D plot of human eye model after GA optimization: (a) $35 \mathrm{~cm}$ and (b) infinite distance.

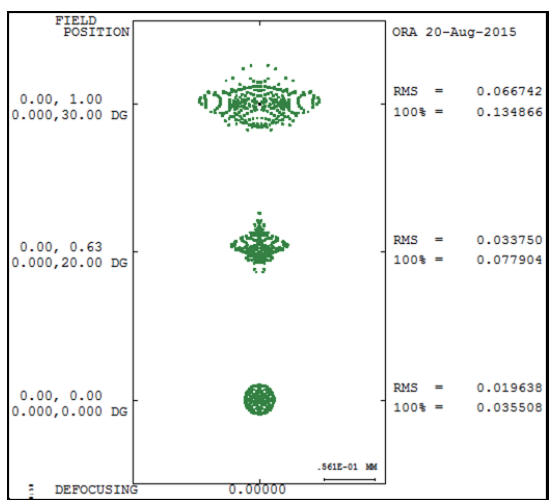

(a)

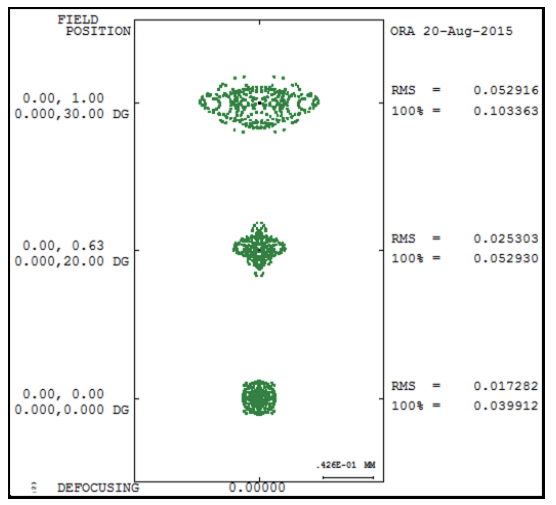

(c)

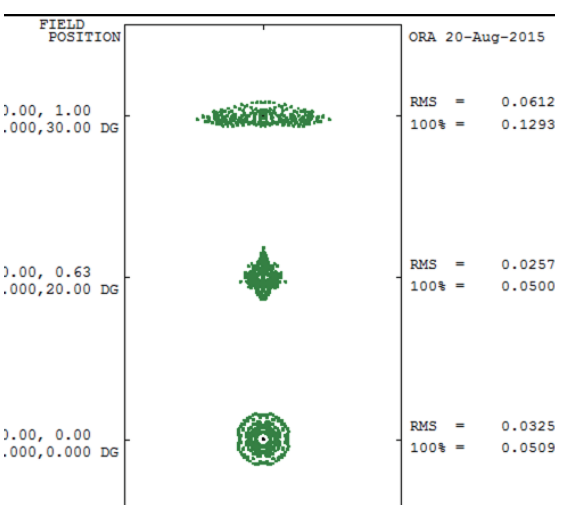

(b)

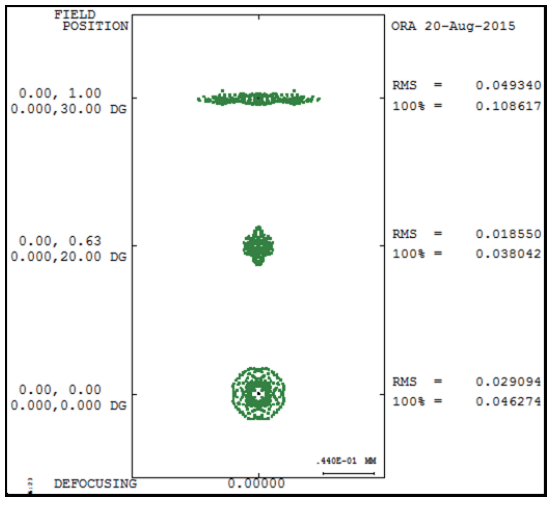

(d)

Fig. 10. (Color online) Spot diagrams of human eye model after CODE V optimization for distances of (a) $35 \mathrm{~cm}$ and (b) infinity, and after GA optimization for distances of (c) $35 \mathrm{~cm}$ and (d) infinity. 


\subsection{MTF performance analysis}

From optical design perspectives, MTF values represent the entire image contrast and sharpness and have a range of 0 to 1 . Generally, images cannot be analyzed after optical design when the MTF value is nearly 0 . To determine the ideal image quality of human eye vision, the MTF must be lower than 0.5 when the spatial frequency is 6 , and the MTF must be approximately 0.3 when the spatial frequency is 15 .

Figure 11 shows MTF plots for the different algorithms and distances. Figures 11(a) and 11(c) show the MTF plots obtained after CODE V and GA optimization for the distance of $35 \mathrm{~cm}$; the MTF values are 74.66 and $77.13 \%$ when the spatial frequency is 15 cycles $/ \mathrm{mm}$, respectively. Figures 11(b) and 11(d) show the MTF plots obtained after CODE V and GA optimization for the infinite distance; the MTF values are 74.46 and $76.1 \%$ when the spatial frequency is 15 cycles/mm, respectively. The results show that higher MTF values are obtained after employing the proposed GA technology.

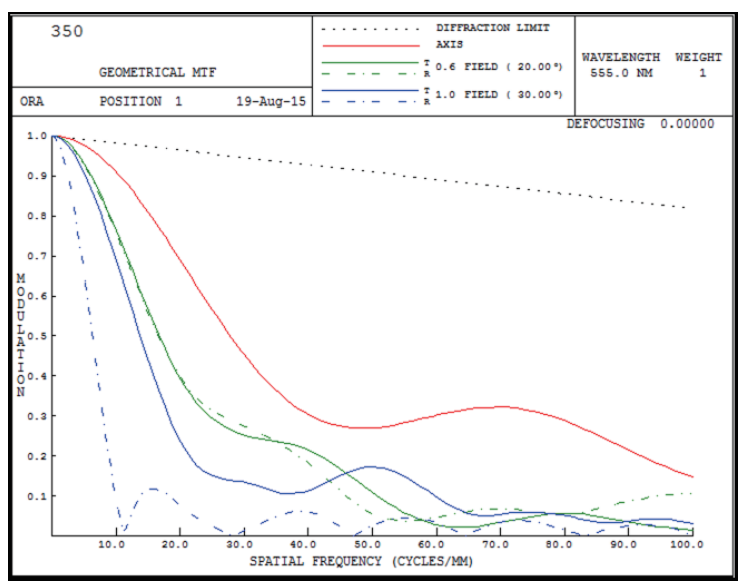

(a)

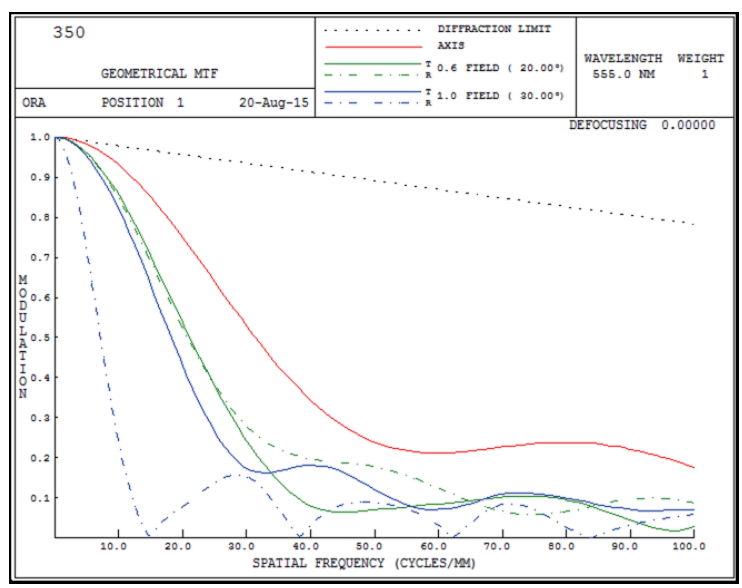

(c)

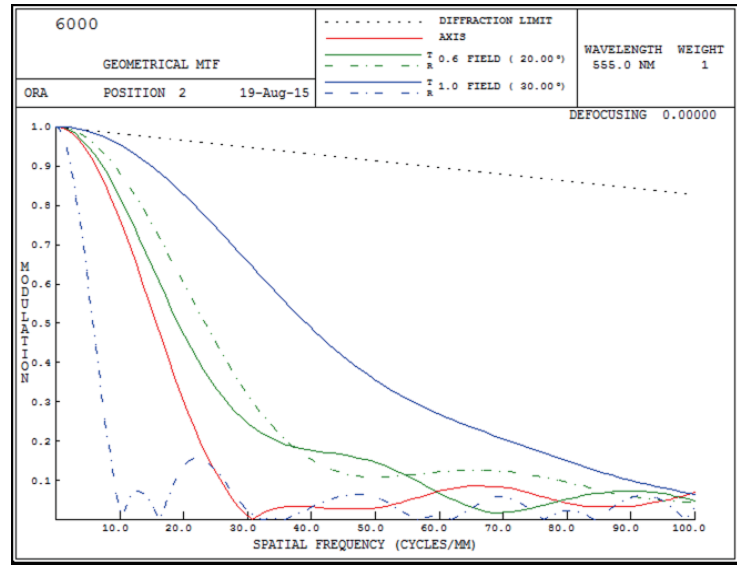

(b)

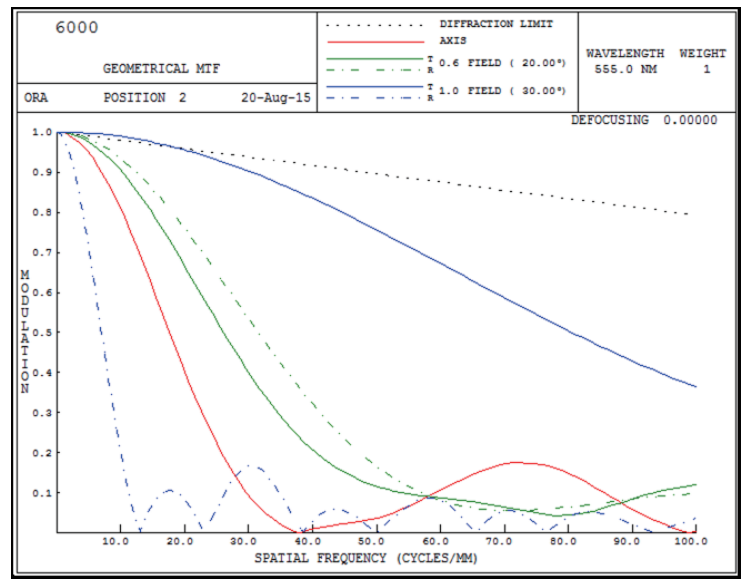

(d)

Fig. 11. (Color online) MTF plots of human eye model after CODE V optimization for distances of (a) $35 \mathrm{~cm}$ and (b) infinity, and after GA optimization for distances of (c) $35 \mathrm{~cm}$; (d) infinity. 


\subsection{Two-dimensional image after applying optical models}

Figure 12 shows the real images passed to the optical models after CODE V and GA optimization. Because the difference in MTF performance between the two algorithms is small, the 2D images passed to the CODE V built-in and GA-optimized optical models are similar (Fig. 12). Regardless of the method used, the images passed through the optimized optical models with the two schemes are all clear.

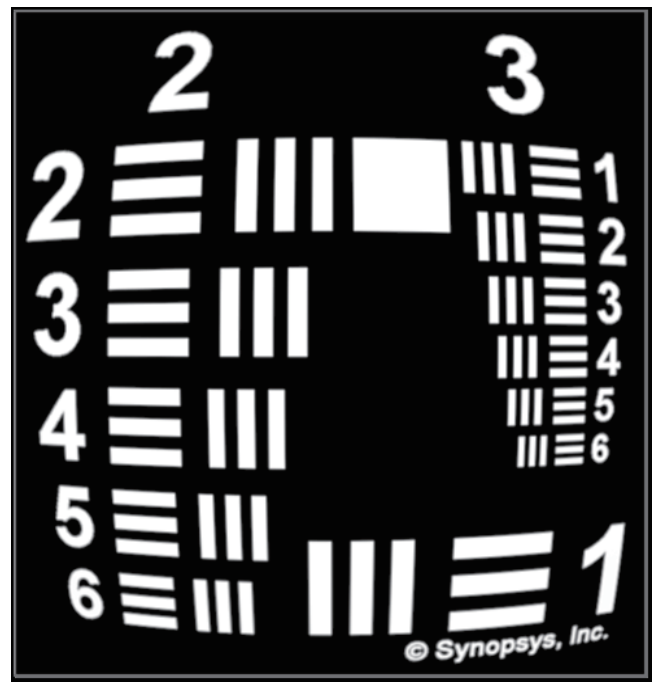

(a)

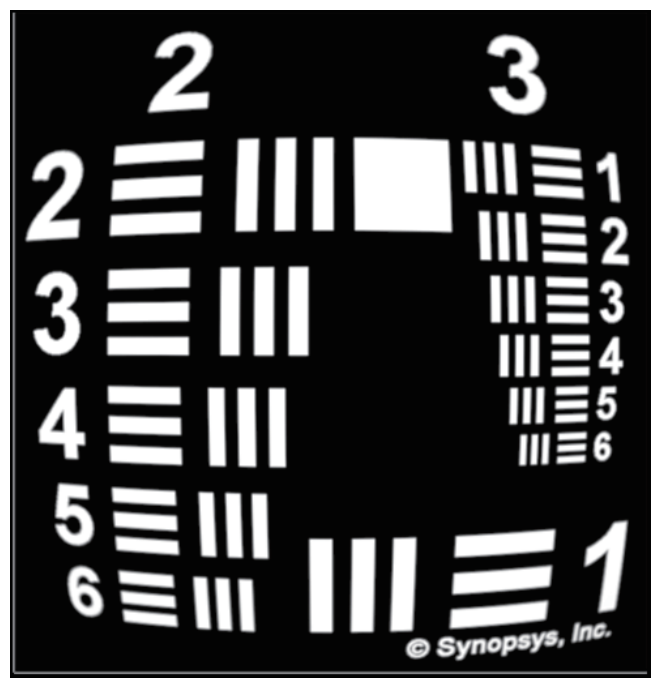

(c)

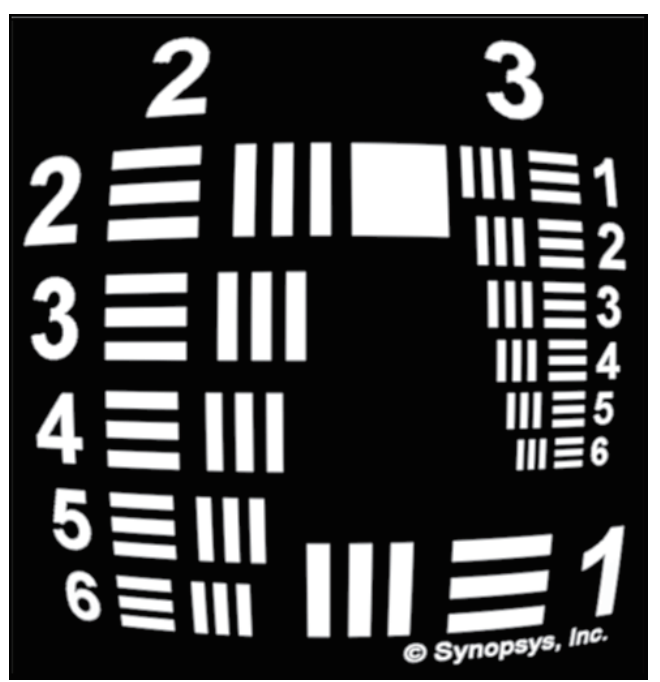

(b)

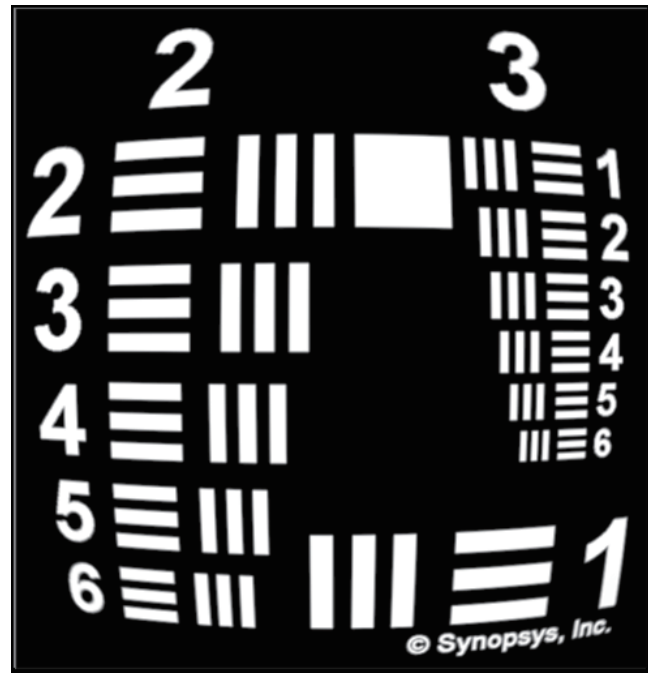

(d)

Fig. 12. 2D image plots of human eye model after CODE V optimization for distances of (a) $35 \mathrm{~cm}$ and (b) infinity, and after GA optimization for distances of (c) $35 \mathrm{~cm}$ and (d) infinity. 


\section{Discussion of Relative Improvements}

The relative improvement of the third-order aberration is given by

$$
\left(\frac{|C O D E V|-|G A|}{|C O D E V|}\right) \times 100 \%
$$

Table 10 shows the third-order aberrations and relative improvements after applying the CODE V built-in algorithm and the proposed GA optimization method for an entrance pupil size of $6 \mathrm{~mm}$. The results show that the proposed GA optimization method is superior to the CODE V built-in algorithm.

The relative improvement of the spot diagram is given by

$$
\left(\frac{C O D E V_{\text {Spot size }}-G A_{\text {Spot size }}}{C O D E V_{\text {Spot size }}}\right) \times 100 \% \text {. }
$$

Table 11 shows the root mean square (RMS) values of the spot diagram and the relative improvements after applying the CODE V built-in algorithm and the proposed GA optimization method for an entrance pupil size of $6 \mathrm{~mm}$. The results show that the proposed GA method is superior to the CODE V built-in algorithm.

Table 10

Third-order aberrations and relative improvements after applying CODE V built-in algorithm and proposed GA

\begin{tabular}{|c|c|c|c|c|}
\hline & SA & $\mathrm{TCO}$ & TAS & SAS \\
\hline \multicolumn{5}{|c|}{ After CODE V optimization } \\
\hline $35 \mathrm{~cm}$ & -0.063012 & 0.132076 & -0.418144 & -0.386417 \\
\hline Infinity & -0.061338 & 0.130273 & -0.388390 & -0.365586 \\
\hline \multicolumn{5}{|c|}{ After GA optimization } \\
\hline $35 \mathrm{~cm}$ & -0.063012 & 0.039395 & -0.396602 & -0.338056 \\
\hline Infinity & -0.060062 & 0.042146 & -0.368300 & -0.319541 \\
\hline \multicolumn{5}{|c|}{ Relative improvement (\%) } \\
\hline $35 \mathrm{~cm}$ & 0 & 71.17 & 5.15 & 12.51 \\
\hline Infinity & 2.08 & 67.64 & 5.17 & 12.59 \\
\hline
\end{tabular}
optimization method for entrance pupil size of $6 \mathrm{~mm}$.

Table 11

RMS values of spot diagram and relative improvements after applying CODE V built-in algorithm and GA optimization method.

\begin{tabular}{lccc}
\hline & CODE V & GA & Relative improvement (\%) \\
\hline $35 \mathrm{~cm}$ & 0.019638 & 0.017282 & 11.99 \\
Infinity & 0.032563 & 0.029094 & 10.65 \\
\hline
\end{tabular}


Table 12

MTF values and relative improvements after applying CODE V built-in algorithm and GA optimization method.

\begin{tabular}{llcc}
\hline & CODE V & GA & Relative improvement (\%) \\
\hline & \multicolumn{4}{c}{ Spatial frequency: 6 cycles $/ \mathrm{mm}$} \\
\hline $35 \mathrm{~cm}$ & 0.966 & 0.974 & 0.8 \\
\hline Infinity & 0.909 & 0.927 & 1.98 \\
\hline & \multicolumn{4}{c}{ Spatial frequency: 15} & cycles $/ \mathrm{mm}$ & \\
\hline $35 \mathrm{~cm}$ & 0.809 & 0.85 & 5.06 \\
\hline Infinity & 0.531 & 0.611 & 15.06 \\
\hline
\end{tabular}

The relative improvement of the MTF is given by

$$
\left(\frac{G A_{M T F}-C O D E V_{M T F}}{G A_{M T F}}\right) \times 100 \% \text {. }
$$

Table 12 shows the MTF values and relative improvements after applying the CODE V builtin algorithm and the proposed GA optimization method for an entrance pupil size of $6 \mathrm{~mm}$ and spatial frequencies of 6 and 15 cycles $/ \mathrm{mm}$. The results show that the proposed GA method is superior to the CODE V built-in algorithm.

\section{Conclusions}

The main conclusion of this study is that the optical design of an IOL (i.e., with improved third-order aberrations, MTF, and spot size for the hyperopia state) using the GA optimization method is advantageous. Compared with the built-in optimization method in CODE V, relative improvements in spot diagram RMS value achieved using the proposed GA scheme were 11.99 and $10.65 \%$ for distances of $35 \mathrm{~cm}$ and infinity in the case of 300 degree hyperopia. Although the improvement of the SA was equal to that for the CODE V built-in optimization method, the crucial aberrations, TCO, TAS, and SAS, were substantially higher. According to the MTF plots for the infinite distance, compared with the CODE V built-in optimization method, the proposed GA optimization method improves the MTF by nearly 1.98 and $15.06 \%$ at spatial frequencies of 6 and 15 cycles $/ \mathrm{mm}$, respectively. In conclusion, the simulation results of third-order aberrations, spot size, and MTF performance show that the proposed GA method for freeform surface lens design can be effectively used in IOL design to enhance image sensing quality.

\section{Acknowledgments}

This study was supported in part by the Ministry of Science and Technology (MOST 108-2221-E-150-022-MY3) and National Taiwan Ocean University.

\section{References}

1 R. D. Binkhorst: Ophthalmic Surg. 6 (1975) 17. https://pubmed.ncbi.nlm.nih.gov/1187085/

2 D. A. Atchison: J. Cataract Refractive Surg. 16 (1990) 178. https://doi.org/10.1016/S0886-3350(13)80727-4 
3 M. Nowakowski: Ph.D. Dissertation, Applied Optics Group School of Physics (National University of Ireland., Ireland, 2011) http://astro.nuigalway.ie/media/collegeofscience/schools/physics/optics/documents/Maciej= Nowakowski PhD thesis.pdf

4 P. Valley, M. R. Dodge, J. Schwiegerling, G. Peyman, and N. Peyghambarian: Opt. Lett. 35 (2010) 2582. https:// doi.org/10.1364/OL.35.002582

5 M. N. Akram and M. H. Asghar: Appl. Opt. 42 (2003) 2312. https://doi.org/10.1364/AO.42.002312

6 Y.-H. Lin, Y.-L. Liu, and G.-D. J. Su: Appl. Opt. 51 (2012) 1804. https://doi.org/10.1364/AO.51.001804

7 G. I. Greisukh, E. G. Ezhov, A. V. Kalashnikov, and S. A. Stepanov: Appl. Opt. 51 (2012) 4597. https://doi. org/10.1364/AO.51.004597

8 B. Chassagne and L. Canioni: Biomed. Opt. Express. 11 (2020) 850. https://doi.org/10.1364/BOE.382162

9 L. Zeng and F. Fang: Appl. Opt. 57 (2018) 7363. https://doi.org/10.1364/AO.57.007363

10 F. Taketani, E. Yukawa, T. Yoshii, Y. Sugie, and Y. Hara: J. Cataract Refractive Surg. 31 (2005) 969. https://doi. org/10.1016/j.jcrs.2004.10.064.

11 P. Pérez-Merino, D. Siedlecki, L. Remón, M. Vinas, J. L. Alió, and J. J. Rozema: Can. J. Ophthalmol. 2020 (2020) 2020. https://doi.org/10.1155/2020/9586062

12 E.-J. Fernandez and P. Artal: IOVS 54 (2013) 838. https://iovs.arvojournals.org/article.aspx?articleid=2151648

13 D. A. Atchison: J Cataract Refractive Surg. 17 (1991) 292. https://doi.org/10.1016/S0886-3350(13)80825-5

14 M. Packer: Expert Rev. Ophthalmol. 6 (2011) 437. DOI: 10.1586/eop.11.40

15 J. Loicq, N. Willet, and D. Gatinel: Proc. 2020 SPIE Design and Quality for Biomedical Technologies XIII Conf. (SPIE 2020) 1123107. https://doi.org/10.1117/12.2544266

16 L. Ji and X. Chang-xi: Acta. Phys. Sin. 47 (2018) 922001-1. https://doi.org/10.3788/gzxb20184709.0922001

17 H.-L. Liou and N. A. Brennan: J. Opt. Soc. Am. A. 14 (1997) 1684. https://doi.org/10.1364/JOSAA.14.001684

18 P. Mukherjee: Ophthalmic Assistants (J. B. M. P., India, 2013) ISBN: 9789350904251

19 J. H. Holland: Adaptation in Natural and Artificial System (MIT P. C., USA, 1992). https://mitpress.mit.edu/ books/adaptation-natural-and-artificial-systems

20 C.-T. Yen and S.-C. Jin: Materials 8 (2015) 6309. https://doi.org/10.3390/ma8095305

21 L. D. Davis, Handbook of Genetic Algorithms (V. N. R., New York, 1991) 1st ed. ISBN-13: 978-0442001735

\section{About the Authors}

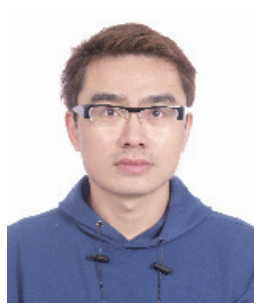

Chih-Ta Yen received his B.S. degree from the Department of Electrical Engineering, Tamkang University, Taiwan, in 1996, his M.S. degree from the Department of Electrical Engineering, National Taiwan Ocean University, Keelung, Taiwan, in 2002, and his Ph.D. degree from the Department of Electrical Engineering, National Cheng Kung University, Taiwan, in 2008. He is currently an associate professor in the areas of communication, optical design, and artificial intelligence technologies at the Department of Electrical Engineering, National Taiwan Ocean University. His major interests are in the areas of multiuser optical communications, machine learning applications, deep learning, image processing, and optical design. He has published over 60 journal papers in the field of electrical engineering. (chihtayen@gmail.com)

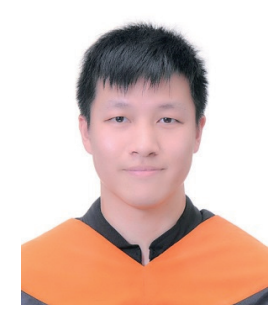

Shih-Cyuan Jin was born in New Taipei City, Taiwan. He received his M.S. degree from the Department of Electrical Engineering, National Formosa University, Taiwan, in 2015. His major interests are in the areas of deep learning, machine learning, and embedded systems. (rupg4fm06@gmail.com) 